\title{
Enhanced Locating Method for Cable Fault Using Wiener Filter
}

\author{
Jeong Jae Han ${ }^{1}$, So Ryoung Park ${ }^{1, *}$, Jeong-Chay Jeon ${ }^{2}$, Taek-Hee Kim², Jae-Gen Yoo ${ }^{2}$ \\ ${ }^{1}$ Department of Information Communications \& Electronics Engineering, The Catholic University of Korea (CUK), Republic of Korea \\ ${ }^{2}$ Electrical Safety Research Institute, Korea Electrical Safety Corporation (KESCO), Republic of Korea
}

Copyright (C) 2015 by authors, all rights reserved. Authors agree that this article remains permanently open access under the terms of the Creative Commons Attribution License 4.0 International License

\begin{abstract}
Time domain reflectometry (TDR) has been widely used for detecting the fault location and fault type of power cable due to the low computational load and good adaptability. However, it is vulnerable in the cable characteristic, such as delay and attenuation fluctuating with frequency. In this paper, we execute the channel estimation using Wiener filter to compute the most similar estimated signal with the injected reference signal, and provide experimental results to show the performance improvement of fault detection and location using TDR with Wiener filter in power cable.
\end{abstract}

Keywords Time Domain Reflectometry (TDR), Correlation Function, Wiener Filter, Gaussian Chirp, Power Cable

\section{Introduction}

There are several locating technologies that can be used to detect the fault location and fault type of power cable. Some of the most publicized methods are time domain reflectometry (TDR), frequency domain reflectometry
(FDR), and time-frequency domain reflectometry (TFDR) [1-6]. TDR method detects the cable fault using the auto-correlation property of the reference signal in time domain. On the other hand, FDR and TFDR consider both of the frequency domain and time domain for detecting the cable fault. The detection error rate of FDR and TFDR methods can be lower than that of TDR method, however FDR and TFDR have higher computational complexity than TDR does [7]. In addition, to overcome the weakness in bandlimited channel and live wire, TDR has adopted various kinds of reference signals such as rectangular pulses, Gaussian enveloped linear chirp, spreading sequences, and modulated spreading sequences with sinusoidal wave.

The reflectometry using auto-correlation property depends on the channel quality critically. Generally in the condition of live wire, the characteristic of channel depends on delay or attenuation fluctuating with frequency. In sort of these cases, it is hard to detect the fault location by conventional TDR method. In this paper, we propose the enhanced locating method for cable fault by reconstructing the injected reference signal from the received (measured) signal. We use the Wiener filter to estimate the channel characteristic and reconstruct the reference signal.

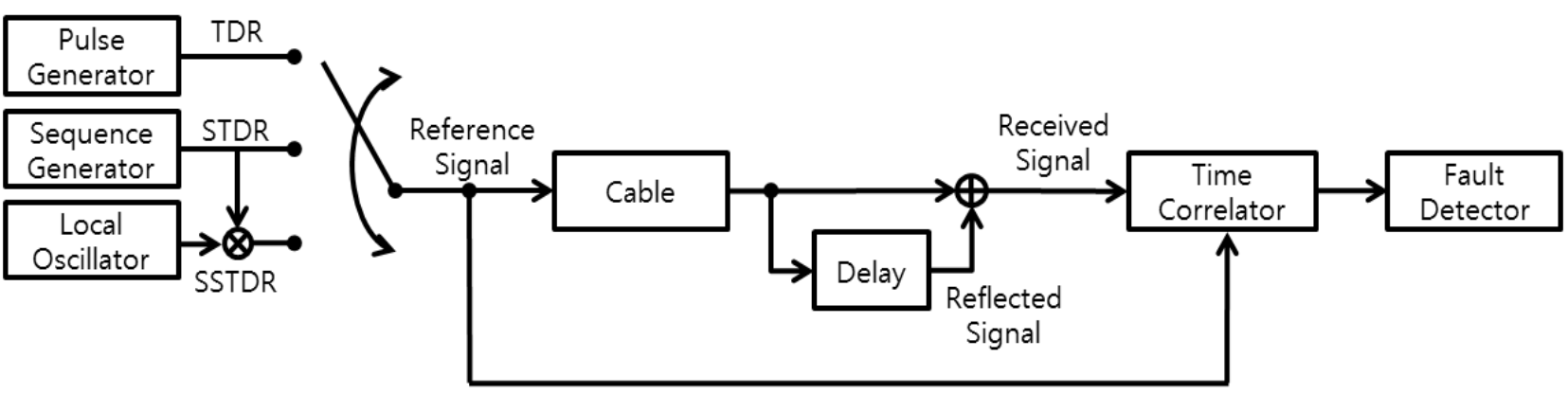

Figure 1. A block diagram of fault detection and location using TDR methods. 


\section{Detection Method and Wiener Filter}

\subsection{Detection Method}

Fig. 1 illustrates the block diagram of TDR methods. The pulse generator makes the rectangular, Gaussian, or raised cosine pulse according to the use. Especially in live wire, in order to avoid the power signal, we can use the Gaussian enveloped linear chirp with specific frequency band. The sequence generator makes the spreading sequences with good auto-correlation property such as $\mathrm{m}$ - or Barker sequence. The sequence TDR (STDR) is the TDR method which is using the spreading sequences to obtain high processing gain. Similarly, spread spectrum TDR (SSTDR) method uses the spreading sequence modulated with sinusoidal wave which is the output of the local oscillator. In TDR, when fault location is near, rectangular pulse which has short pulse width can be suitable. On the other hand, when fault location is far, the amplitude attenuation is very large and different with frequency band, hence the rectangular pulse is not appropriate for reference signal anymore. In the case of far fault, Gaussian chirp can be generally suitable for waveform of reference signal. The advantage of Gaussian chirp is both that we can deploy the Gaussian chirp to desired frequency band and that we can handle the bandwidth of Gaussian chirp by adjusting frequency acceleration. Therefore we can modify Gaussian chirp as the effective reference signals for many different channels [8]. The measured signal from the cable (including the distorted reference signal, and any reflections observable at the receiver) is fed into a time-correlator along with a reference signal. We can find the fault location and type by analyzing the time of arrival (TOA) and phase of reflected signal obtained from time correlation. The distance $d$ from signal injecting point to fault location is able to be calculated by Eq. (1) where $v_{p}$ is velocity of propagation (VOP) of reference signal and $\tau_{D}$ is the time difference between the reference signal with the reflected signal [1-2].

$$
d=\frac{v_{p} \times \tau_{D}}{2}
$$

\subsection{Wiener filter}

Fig. 2 illustrates a simplified block diagram of Wiener filter. A finite impulse response (FIR) filter produces the minimum mean-square estimate of a injected reference signal $x(n)$ by filtering a received signal $y(n)$ with Wiener filter $W=[w(0), w(1), \ldots, w(p)]$. It is assumed that $y(n)$ and $x(n)$ are jointly wide-sense stationary with known auto-correlations. We can calculate Wiener filter coefficient vector using $\boldsymbol{r}_{x y}$ and $\boldsymbol{R}_{y}$ using Eq. (2), where $\boldsymbol{r}_{x y}$ is the cross-correlation vector of reference signal with received signal and $\boldsymbol{R}_{\boldsymbol{y}}$ is the auto-correlation matrix of received signal [9].

$$
\mathbf{w}=\boldsymbol{R}_{y}^{-1} \boldsymbol{r}_{x y}
$$

Also we can describe the $z$-transformed $p$-th order Wiener filter on $Z$-domain as Eq. (3).

$$
W(z)=w(0)+\sum_{i=1}^{p} w(i) z^{-i}
$$

We can calculate the optimized Wiener filter coefficients for channel by finding the order $p$ which produces the minimum error $e(n)$ between estimated reference signal $\hat{x}(n)$ and reference signal $x(n)$. The estimated reference signal $\hat{x}(n)$ can be calculated by convolution between Wiener filter coefficient $w(n)$ and received signal $y(n)$. Hence estimated reference $\hat{x}(n)$ which has the minimum $e(n)$ can be produced. Fig. 3 describes fault detection method which applies Wiener filter.

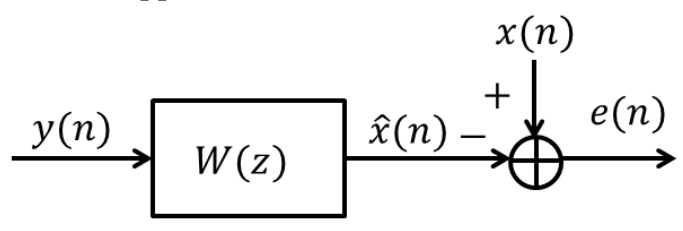

Figure 2. A simplified block diagram of Wiener filter.

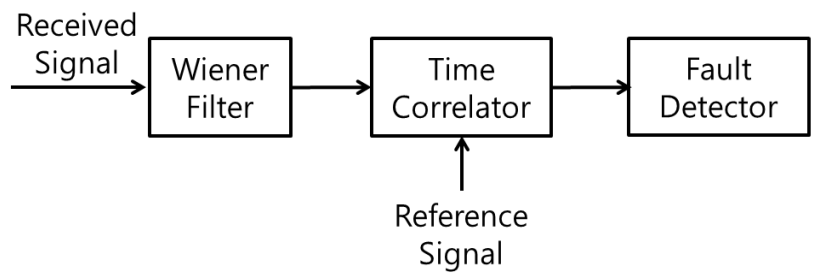

Figure 3. A block diagram of fault detection method using Wiener filter

\section{Experimental Results}

This chapter contains the performance improvement of the fault detection when the Wiener filter is used for detection or not. We have performed signal processing for experimental results using $\mathrm{MATLAB}^{\circledR}$ and used the rectangular pulse (length: 4 , amplitude: 1 ) on dead wire and Gaussian chirp signal (length: 35, amplitude: 1) on live wire for the injected signals respectively. We set the sampling frequency $f_{s}=208.3[\mathrm{MHz}]$ and the measured VOP of power cable $v_{p}=1.911 \times 10^{8}[\mathrm{~m} / \mathrm{s}]$. Both of these sampling frequency and VOP are obtained as best experimental property at KESCO. The experiments are implemented from measured data of commercial power line cable (CV_2C_6SQ). Every physical data about 3 fault types ('open', 'partial disconnection', and 'insulation damage') and various distances are gained from KESCO. The upper part of figures shows the time correlation of the reference signal and received signal without Wiener filtering, otherwise the bottom of figures show time correlation of the reference signal and the output signal from the Wiener filter. In here, we set the orders of Wiener filter as long as length of the reference signals.

Fig. 4 shows the detection performance when the injected signal is rectangular pulse, the fault location is $160 \mathrm{~m}$ from signal injecting point, and the fault type is 'open' on dead wire. It is shown that the fault detection error with using Wiener filter is smaller than result without using Wiener filter. It is confirmed that Wiener filtering is improving the detection performance. 

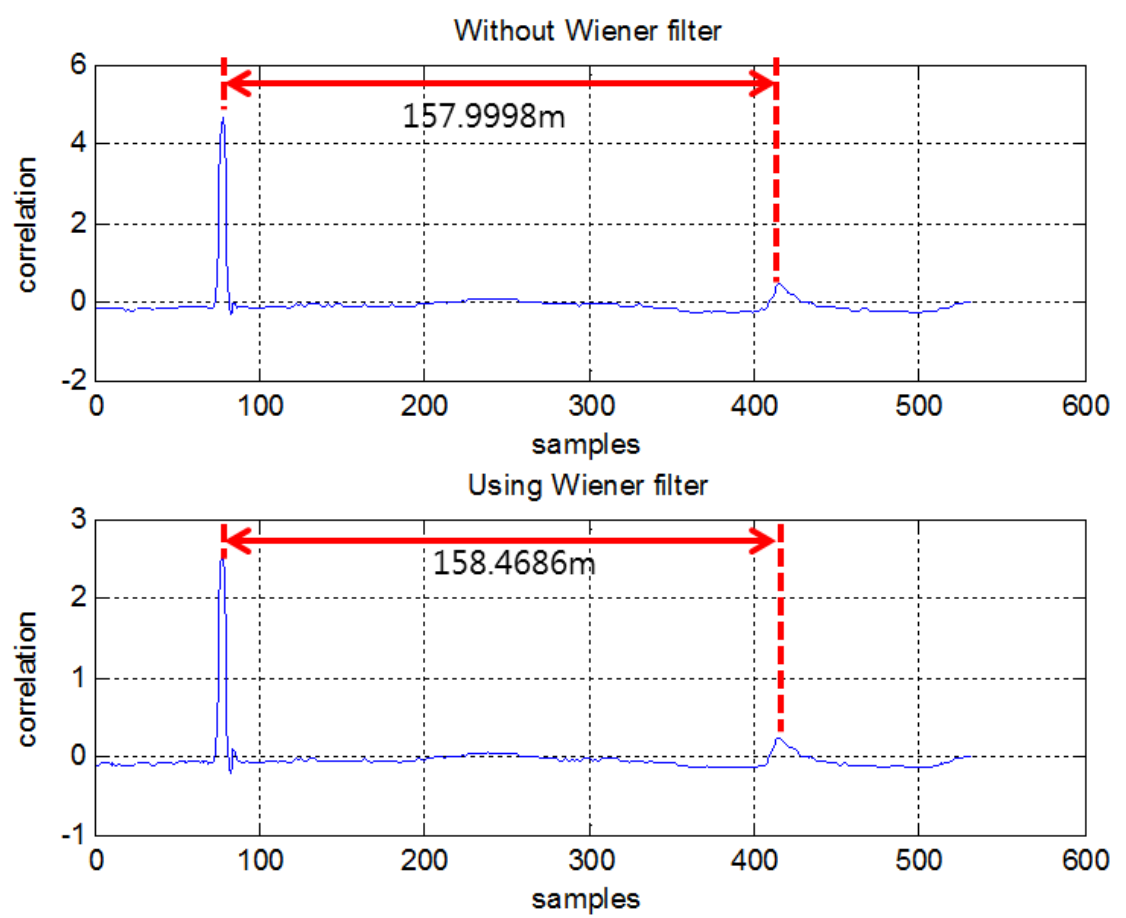

Figure 4. The detection performances (reference signal: rectangular pulse, fault location=160m, fault type: open, 20-th order Wiener filter)

Fig. 5 shows the detection performance when the injected signal is the Gaussian chirp, the fault location is $23.45 \mathrm{~m}$, and the fault type is 'open' on live wire. Both methods whether using Wiener filter, implemented almost the same performance. When fault location is near, attenuation portion is small also fault type 'open' does not distort reference seriously. Thus performance improvement is negligible when Wiener filter is adopted or not.

Fig. 6 shows the results when reference signal is Gaussian chirp, fault location is $27 \mathrm{~m}$ and fault type is 'partial disconnection'. It is shown that the method using Wiener filter provides better performance compare with the method without Wiener filter. Thus, it is confirmed clearly that the fault detection method using Wiener filter is effective to improving the detection performance.
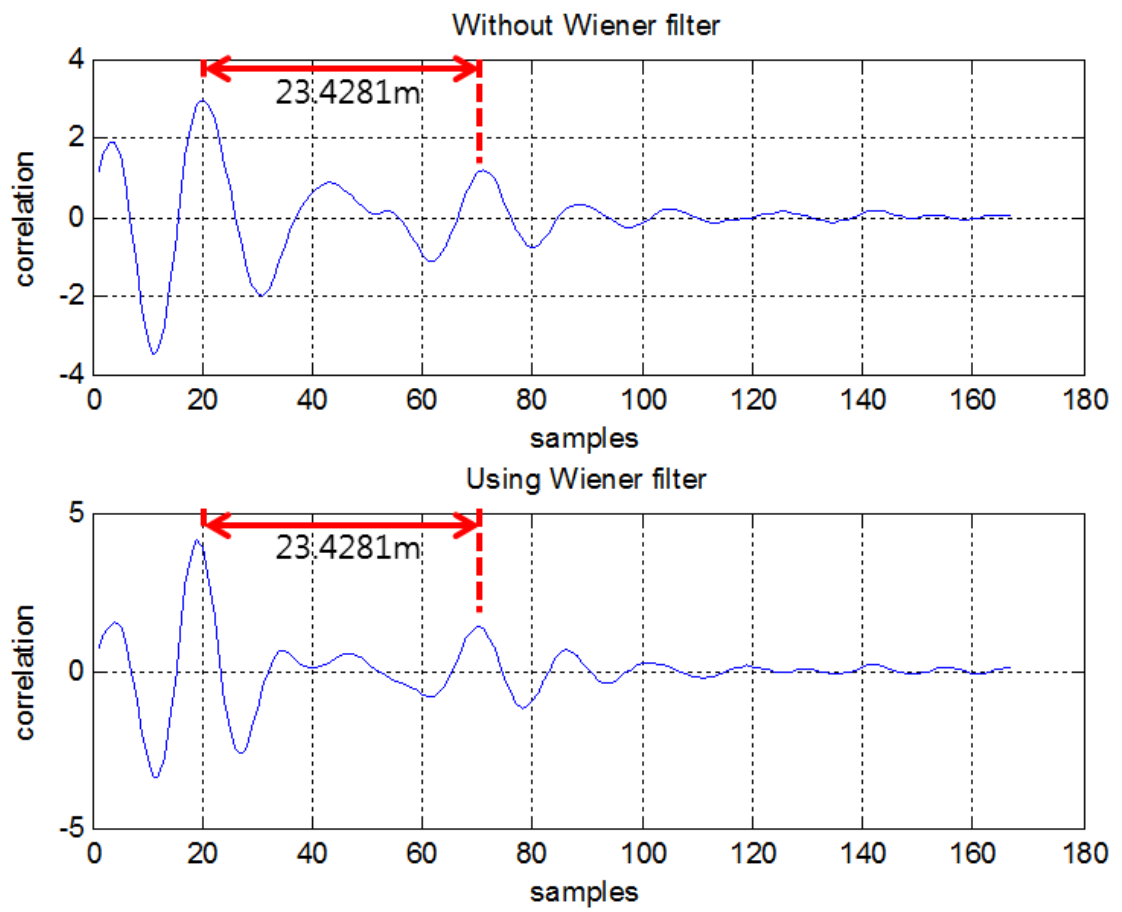

Figure 5. The detection performances (reference signal: Gaussian chirp signal, fault location=23.45m, fault type: open, 35 -th order Wiener filter) 

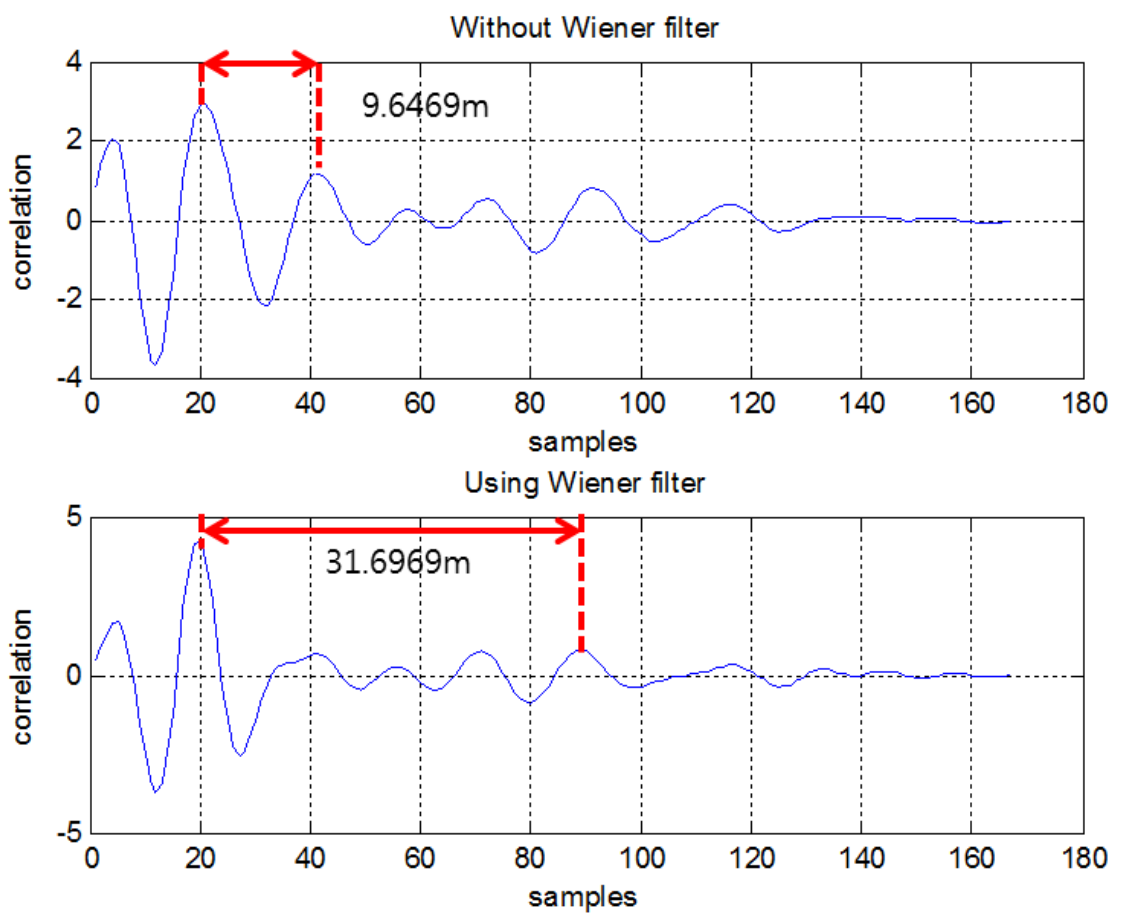

Figure 6. The detection performances (reference signal: Gaussian chirp signal, fault location=27m, fault type: partial disconnection, 35-th order Wiener filter)

Fig. 7 shows the experimental results when the reference signal is Gaussian chirp, the fault location is $23.45 \mathrm{~m}$ and the fault type is 'insulation damage'. It is shown that side-lobes are decreased when Wiener filter is adopted, hence estimated fault location is changed from $17.4563 \mathrm{~m}$ (without using Wiener filter) to $24.3469 \mathrm{~m}$ (using Wiener filter). It is obvious that using Wiener filter is able to improve fault detection performance.
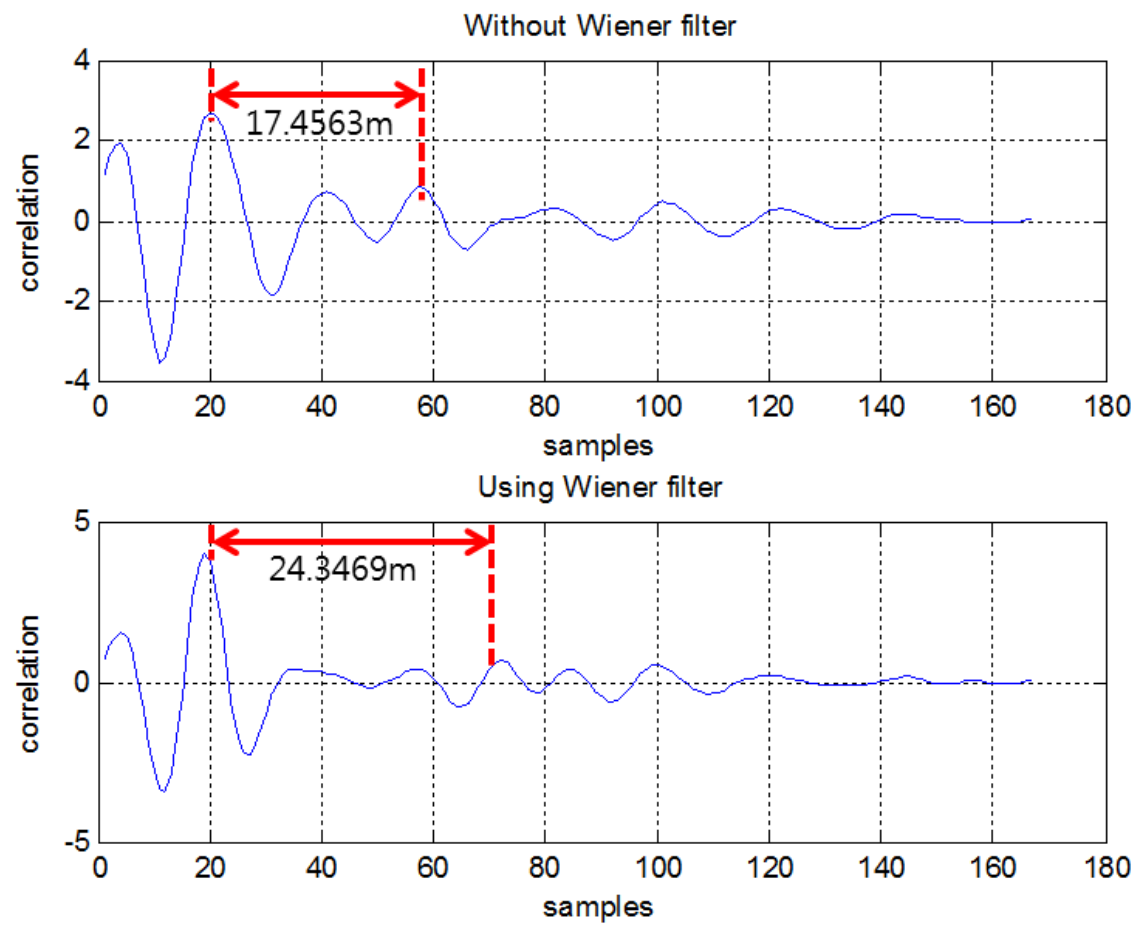

Figure 7. The detection performances (reference signal: Gaussian chirp signal, fault location $=23.45 \mathrm{~m}$, fault type: insulation damage, 35 -th order Wiener filter) 


\section{Conclusions}

This paper has examined TDR which is one of detection method for the fault location and type in power cable, and Wiener filter to overcome the channel characteristic such as delay and attenuation which influence bad effect for fault detection performance. Also we have identified fault detection performance whether using Wiener filter with measured data from commercial power line cable. Most of simulation results illustrate detection method using Wiener filter has less error than that without Wiener filter. Consequently, we can confirm the performance of fault detection method using Wiener filter is better than that without Wiener filter.

\section{Acknowledgments}

This study was supported by "2013 Dual Use Technology Program".

\section{REFERENCES}

[1] R. A. Guinee, "A novel transmission line test and fault location methodology using pseudorandom binary sequences," in Proc. IET Irish Conf. Sign. Syst., pp. 350-355, Galway, Ireland, Jun. 2008.

[2] J. J. Han and S. R. Park, "Performance comparison and improvement of STDR/SSTDR applying various sequences," J. Korean Inst. Commun. Inf. Sci. (KICS), vol. 39, no. 11, pp. 637-644, Nov. 2014.

[3] S. H. Doo, K. S. Kwak, and J. B. Park, "Estimation of fault location on power line using the time-frequency domain reflectometry," J. Inst. Electron. Inf. Eng. (IEIE), vol. 57, no. 2, pp. 268-275, Feb. 2008.

[4] C. K. Lee, T. S. Yoon and J. B. Park, "Localization of concentric neutrals corrosion on live underground power cable based on time-frequency domain reflectometry," J. Inst. Electron. Inf. Eng. (IEIE), vol. 62, no. 2, pp. 239-245, Feb. 2013.

[5] P. Smith, C. Furse, and J. Gunther, "Analysis of spread spectrum time domain reflectometry for wire fault location," IEEE Sensor J., vol. 5, no. 6, pp. 1469-1478, Dec. 2005.

[6] J. J. Han, S. Noh, and S. R. Park, "Improvement of detection performance in STDR/SSTDR using sign detector," Electronics Letters, vol. 51. (Accepted for publication on July 2015)

[7] Y. J. Shin, E. j. Powers, T. S. Choe, C. Y. Hong, E.S Song, J.G. Yook and J.B. Park, "Application of time-frequency domain reflectometry for detection and localization of a fault on a coaxial cable," J. IEEE Trans. Instrum. Meas., vol. 54, no. 6 , pp. 2493-2500, Dec. 2005.

[8] J. C. Hong, K. H. Sun, and Y. Y. Kim, "Wave damage detection by the matching pursuit using the Gaussian chirp pulses," in Proc. IEEE Int. Ultrasonics Ferroelectrics and Frequency Control Joint $50^{\text {th }}$ Anniversary Conf., vol. 3, pp. 2310-2313, Aug. 2004.

[9] Monson H. Hayes, Statistical Digital Signal Processing and Modeling, 1st Ed., Wiley, 1996, p.337. 